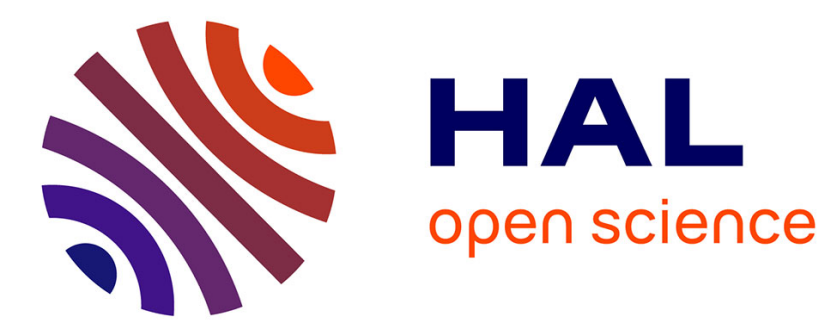

\title{
Évolution de processus autistiques au travers du psychodrame psychanalytique à médiations
}

\author{
Laurence Barrer
}

\section{To cite this version:}

Laurence Barrer. Évolution de processus autistiques au travers du psychodrame psychanalytique à médiations. Revue de psychothérapie Psychanalytique de Groupes, 2010, 55 (2), 10.3917/rppg.055.0089 . hal-01774727

\section{HAL Id: hal-01774727 \\ https://hal.science/hal-01774727}

Submitted on 23 Apr 2018

HAL is a multi-disciplinary open access archive for the deposit and dissemination of scientific research documents, whether they are published or not. The documents may come from teaching and research institutions in France or abroad, or from public or private research centers.
L'archive ouverte pluridisciplinaire HAL, est destinée au dépôt et à la diffusion de documents scientifiques de niveau recherche, publiés ou non, émanant des établissements d'enseignement et de recherche français ou étrangers, des laboratoires publics ou privés. 


\title{
ÉVOLUTION DE PROCESSUS AUTISTIQUES AU TRAVERS DU PSYCHODRAME PSYCHANALYTIQUE À MÉDIATIONS
}

\author{
Laurence Barrer
}

ERES | Revue de psychothérapie psychanalytique de groupe

$2010 / 2-n^{\circ} 55$

pages 89 à 101

ISSN 0297-1194

Article disponible en ligne à l'adresse:

http://www.cairn.info/revue-de-psychotherapie-psychanalytique-de-groupe-2010-2-page-89.htm

Pour citer cet article :

Barrer Laurence, «Évolution de processus autistiques au travers du psychodrame psychanalytique à médiations », Revue de psychothérapie psychanalytique de groupe, 2010/2 n55, p. 89-101. DOI : 10.3917/rppg.055.0089

Distribution électronique Cairn.info pour ERES.

(c) ERES. Tous droits réservés pour tous pays.

La reproduction ou représentation de cet article, notamment par photocopie, n'est autorisée que dans les limites des conditions générales d'utilisation du site ou, le cas échéant, des conditions générales de la licence souscrite par votre établissement. Toute autre reproduction ou représentation, en tout ou partie, sous quelque forme et de quelque manière que ce soit, est interdite sauf accord préalable et écrit de l'éditeur, en dehors des cas prévus par la législation en vigueur en France. II est précisé que son stockage dans une base de données est également interdit. 


\section{ÉVOLUTION DE PROCESSUS AUTISTIQUES AU TRAVERS DU PSYCHODRAME PSYCHANALYTIQUE À MÉDIATIONS}

LAURENCE BARRER

Le psychodrame psychanalytique de groupe d'enfants très régressés fait aujourd'hui partie des champs à explorer, tant au niveau de l'aménagement particulier qu'il impose, qu'aux apports évolutifs indéniables pour les enfants au travers de la reprise du développement de la chaîne associative de groupe. Je montrerai ici comment la création d'un cadre particulier avec médiateurs a permis à un enfant autiste de transformer les processus autistiques qui l'envahissaient. L'adaptation du dispositif, où nous avons considéré les agirs comme des préfigurations de symbolisation sous-tendant la transformation de processus psychiques archaïques en primaires, puis secondaires, a joué le rôle de contenant de ces mouvements déstructurants. Préfiguration au sens où l'agir, comme l'expression corporelle, est signe pour nous d'un irreprésentable qui peut advenir. Un des médiateurs, sous les caractéristiques d'un objet concret réel et de ses métamorphoses, fit relation entre les membres. L'objet concret externe, de l'expression de la symbolique de l'enveloppe individuelle, amena le groupe psychodramatique à élaborer la symbolique paternelle, dans un mouvement synergique de ces différents éléments, aller-retour entre groupe et individu.

Le psychodrame psychanalytique à médiations eut lieu en 2002 dans un hôpital de jour. Avant l'ouverture du groupe, l'institution était aux prises avec des dysfonctionnements majeurs. Depuis sa création, il y avait eu un «turn-over» des soignants et des soignés. L'institution n'avait jamais travaillé avec des groupes fermés et l'activisme était la règle pour lutter contre les attaques psychotiques des soignés liées à la 
pathologie, et, celles de la hiérarchie aux prises avec une autre forme de déliaison. La conséquence sur le groupe a été une difficulté à maintenir un rythme régulier d'une fois par semaine de septembre à juin. Le cadre a été malmené et seules vingt séances ont eu lieu (institution fermée pour vacances scolaires, enfants du psychodrame partis en camps thérapeutiques, RTT et maladies). Ainsi, quatre séances en février et cinq consécutives en mai n'ont pas eu lieu. Mais, malgré ces conditions difficiles, les trois patients se sont appropriés l'espace et, au travers de la chaîne associative du groupe, ont re-lancé leur questionnement. Au niveau de mon contre-transfert, le manque de continuité temporelle du groupe est resté actif pendant la préparation de mon texte où je me suis trompée par deux fois de timing mettant en péril ma présentation orale au colloque, puis en mélangeant les âges, les mois et les années des enfants.

\section{DISPOSITIF DU PSYCHODRAME PSYCHANALYTIQUE À MÉDIATIONS}

La technique du psychodrame psychanalytique de groupe a été notre base, mais nous y avons ajouté deux supports concrets pour ces patients dont l'imaginaire était très pauvre. Un enfant autiste était nonverbal, le deuxième post-autiste était collé au concret et le troisième, diagnostiqué psychotique, apportait les images d'un monde morcelé et violent. L'élaboration de scénarii en commun sur un mode verbal était compromise. Nous avons donc aussi privilégié le non-verbal. Nous avons aussi pris en compte ce que Bion énonce comme « un bon esprit de groupe $»^{1}$ à propos de la résolution de tension interne au groupe, ses limites, sa contenance...

\section{Les médiateurs}

Un conte était lu au groupe en début de séance pour inciter à la représentation imaginaire et à la projection dans un personnage.

Le deuxième médiateur était une boîte de déguisements qu'on ouvrait après la lecture et la distribution des rôles ; nous avions imaginé amener le groupe, dans un second temps, à la figuration du faire semblant en partant du concret, de la sensorialité du vêtement, de là où ils en étaient, à la secondarisation de leurs processus. L'hypothèse sous-tendue par ces deux médiateurs comme support, était de leur indiquer symboliquement le « comment jouer », prendre un rôle, le jouer en s'appuyant chacun sur ses objets internes. 


\section{Les règles}

Elles étaient énoncées à la première séance, puis rappelées dès qu'elles étaient transgressées.

- L'association libre : "Ici on dit tout ce qu'on a envie de dire, même des gros mots pour en parler. » Nous avions rajouté les « gros mots» pour leur signifier qu'il n'y avait pas de sanction ici dans le dire. Notre hypothèse était qu'en acceptant leurs paroles, ils pourraient ainsi jouer spontanément sans retenue. Ils pouvaient tout dire.

- La confidentialité : "Ce qui est dit dans la pièce de psychodrame reste dans la pièce, on ne racontera rien aux parents ou aux autres personnes de l'institution. » Leur défaut de pare-excitation et de construction psychique ne garantissait pas la confidentialité de leur place, mais de la nôtre nous l'annoncions en utilisant des mots à leur portée.

- La restitution n'a pu être mise en place totalement ; de part leur pathologie, ils ne faisaient pas de lien entre les différents espaces et un enfant ne parlait pas au début du groupe.

- L'abstinence n'a pas pu être respectée puisque les enfants étaient toute la journée ensembles dans l'institution. Ici, je me suis posée la question de la vue d'Anzieu sur ce point ${ }^{2}$ où il associe la règle d'abstinence à la règle de faire semblant. « La règle d'abstinence se particularise au psychodrame en règle de faire semblant », pour lui, elle implique que "psychodramatisants et psychodramatistes ont à s'abstenir de satisfaire directement entre eux leurs pulsions sexuelles et agressives ». Ceci paraît relever du pacte dénégatif (des négatifs) définit par Kaës qu'il nous semble effectivement important de ne pas révéler. Anzieu interroge aussi ici le corporel. Dans les groupes d'enfants très régressés, il n'est pas possible de respecter la règle du non-toucher entre patients et cothérapeutes. Quand un enfant autiste fait une crise de tantrum en séance, il est important de le contenir avec notre corps afin de lui permettre de se rassembler pour qu'il puisse continuer le groupe. $\mathrm{Et}$, comme on le verra plus loin, les mouvements archaïques appellent le sensoriel ou inversement. Nous avions énoncé la règle du faire-semblant et de l'interdit du toucher : «On ne se frappe pas, on ne se touche pas, on fait semblant, on ne se fait pas mal, ni à soi, ni aux autres (et le cadre) ; on va se rencontrer une fois par semaine ici pour jouer à faire semblant. » Et par cette règle, ils ne pouvaient tout faire.

La règle de l'abstinence garantissait l'intégrité corporelle de chacun en espérant aboutir à un travail métaphorique - passage de la matière à la pensée -, de créer un espace, et de symboliser l'interdit de l'inceste. La configuration incestuelle des espaces familiaux était tellement présente dans leurs histoires respectives.

Nous avons énoncé deux règles spécifiques à ce groupe : 
- Le non-perçage de l'enveloppe du groupe contre les angoisses de vidage. " On ne sort pas de la salle avant la fin du jeu, vous buvez et allez aux toilettes avant d'entrer ». Le défaut d'enveloppe, de contenant, d'indifférenciation entre intérieur et extérieur était tel qu'ils pouvaient entrer et sortir impulsivement n'importe quand. Nous devions donc être le garant de l'enveloppe salle de psychodrame.

- La contenance des pulsions liées au pacte dénégatif. «Si vous ne respectez pas les règles du groupe, on vous sort du groupe, vous serez assis entre nous, hors-jeu, jusqu'à ce vous soyez calmés pour retourner dans le groupe. » Nous voulions instaurer une possibilité de couper dans le pulsionnel sexuel ou de violence physique au cas où ils ne puissent le faire d'eux-mêmes. Avec, en arrière-fond, le désir de leur faire comprendre que le groupe, reflet social, n'accepte pas le pulsionnel. Avec la répétition du mot « groupe » ici, nous voulions marquer quelque chose d'une appartenance au groupe et non de la domination de l'adulte sur l'enfant, de la prévalence d'une asymétrie.

\section{Le dispositif}

Dans le psychodrame psychanalytique de groupe, l'espace et le temps sont découpés pour topographier les lieux psychiques par rapport aux lieux physiques. Nous avons gardé la différenciation en organisant cinq chaises en cercle dans une partie de la salle et l'autre partie pour jouer. Nous avons été obligées de mettre sur le côté les chaises des enfants suite à l'utilisation de l'une d'elles comme fronde. Le temps a été séparé en quatre, trois pour le jeu psychodramatique et un temps d'une heure pour les cothérapeutes à la fin du groupe pour écrire et discuter.

Le premier temps psychodramatique était de 5-10 minutes, temps d'élaboration du thème, où tous assis, la cothérapeute ou un patient, s'il le souhaitait, lisait le conte. Puis on demandait quel rôle ils voulaient jouer et de décrire un minimum ce que ferait le personnage. Nous avions précisé qu'ils pouvaient nous donner un rôle. On sollicitait les enfants qui n'avaient pas accès à la parole en se saisissant d'un mot lancé, d'une émotion ou d'un comportement non-verbal, éléments $\beta$ expulsés, en les transformant en rôle. On pouvait supposer qu'il jouait M. Rien par exemple. Comme si nous étions des traducteurs de leur expression inconsciente, nous réceptionnions au travers de notre fonction $\alpha$, ces éléments bruts, archaïques que nous transformions en sens pour le groupe et a fortiori pour le patient. Bion théorise « un concept de fonction-alpha comme un instrument de travail dans l'analyse des troubles de la pensée $»^{3}$. Il évoque l'identification projective de l'enfant vers la mère qui va transformer la projection grâce à sa fonction $\alpha$. Parallèlement, nous tentions de transformer les éléments projetés du

3. W. R. Bion, Réflexion faite, Paris, PuF, 1983, p. 131-133. 
groupe en sens commun pour être travaillés dans le groupe. M. Rien qui ne faisait rien, annoncera bien des séances plus tard qu'il jouera le rôle du père.

Le deuxième temps était celui du jeu d'une demi-heure. On ouvrait la boîte de déguisements pour matérialiser le «faire semblant» comme pour se mettre dans la peau d'un autre. Les enfants prenaient le vêtement de leur choix, remis en commun à chaque séance. Comme le vêtement symbolisait le rôle, nous pensions traduire le possible changement de peau, de place à venir et avenir, en matérialisant ainsi la fluidité des positions psychiques. Les enfants devaient aussi le laisser dans la salle mais n'étaient pas obligés le de ranger. Les cothérapeutes le faisaient pendant que les enfants étaient en train de sortir. Nous nous différencions ainsi de l'éducatif. Je pense que cet élément clinique dans les psychodrames suivants a été aussi le symbole de la fonction contenante du groupe, où les vêtements/états psychiques ont été rangés/ protégés par les adultes/thérapeutes. D'ailleurs dans les psychodrames suivants, des petits patients ont voulu participer à ce rangement, mais c'est une autre histoire...

Le troisième temps était celui de la discussion du jeu avec ou sans déguisement en reformant le cercle. Ainsi, ils pouvaient parler de leur place (sans déguisement) ou de la place du personnage (si la transition entre le jeu et la restitution était trop soudaine, cela leur laissait le temps de se poser). Ce temps a varié, d'aucun rendu les premières séances, à dix minutes pour les dernières.

\section{Les participants}

Dans ce deuxième psychodrame de septembre à juin, nous étions deux cothérapeutes, une infirmière de l'institution et moi-même. Les années suivantes, $\mathrm{j}$ 'ai introduit une troisième co-thérapeute pour être l'observatrice-scribe du groupe. Les conflits institutionnels s'étaient apaisés et d'autres soignants étaient intéressés par le psychodrame. Ici, nous avions décidé d'en faire bénéficier quatre patients, mais un patient sortit précipitamment de l'hôpital avant la première séance (sortie indépendante du psychodrame). Les plannings de l'institution étant faits, nous n'avons pas pu prendre un autre enfant.

Le premier enfant, Antoine (5 ans et demi), autiste, était un enfant du placard. La protection de l'enfance l'avait sorti à 4 ans d'un appartement sans lumière du jour où il vivait avec une grand-mère aveugle, obsessionnelle, et un père paranoïaque. Attaché pour manger, il passait ses journées enfermé dans une chambre, entouré de sachets plastiques lui servant de « jouet » devant la télévision. Il n'avait pas revu sa mère qui séjournait souvent en psychiatrie pour dépression entrecoupée de multiples tentatives de suicide suivies de mois de comas. Chaque membre de la famille était enfermé dans sa pathologie et coupé du monde. 
Antoine était le deuxième d'une fratrie de trois, tous placés jeunes à cause de la violence physique du père. Son frère aîné était aussi son cousin, puis la mère eut une liaison avec un ami de son troisième compagnon et mit une fille au monde. L'oncle d'Antoine qui est, je le rappelle, le père du frère aîné, sera incarcéré pour infanticide sur son nourrisson de quelques mois. La configuration familiale était extrêmement trouble avec des mouvements incestueux.

À son entrée dans l'hôpital, Antoine ne prononçait que des syllabes, ne jouait pas, n'écrivait pas. Il trottinait sur la pointe des pieds, un sachet plastique à la main volant derrière lui. Il ne s'adressait qu'à l'adulte et lui imposait sa volonté en utilisant sa main. Nous voulions travailler sur la relation à l'autre et faire évoluer la relation d'objet en lui servant de prothèse du Moi par étayage sur le groupe de psychodrame.

Alain (8 ans et demi), psychotique, était né prématurément à 6 mois par césarienne. Séparé de sa mère, il était allé d'hôpital en hôpital, les médecins avaient dit à la mère qu'il ne vivrait pas. La famille partit quatre ans à l'étranger et revint en France. La mère était très dépressive, peu affective, et le père dit infantile. Alain avait deux sœurs plus âgées qui s'occupaient de lui comme d'une poupée. Il avait un sac de petites figurines dont il ne pouvait se séparer. Ses histoires n'étaient que du coq à l'âne. Il restait seul ou contrôlait les enfants comme des marionnettes. On souhaitait travailler avec lui au travers du groupe sur la différenciation des espaces et élaborer une relation d'objet moins fusionnelle, de tenter de passer des représentations de chose aux représentations de mot.

Kenny (8 ans et demi), diagnostiqué autiste petit, était en relation et parlait bien, mais il gardait des comportements autistiques. Depuis son placement chez une assistante familiale, suite à des maltraitances physiques à 3 mois avec son frère aîné de 2 ans, il continuait à voir son père, mais sa mère avait complètement disparu. L'assistante familiale était très fusionnelle. Nous avions décidé de son entrée dans le groupe au vu de son manque d'investissement narcissique. Il était collé à l'adulte qu'il accaparait, qu'il vampirisait et aux autres enfants qui le sadisaient.

\section{LE PSYCHODRAME PSYCHANALYTIQUE À MÉDIATIONS}

Je vais rapporter ici les premières séances où un objet concret externe, une salopette eut différentes fonctions reflétant ainsi l'évolution des états psychiques du groupe et d'un patient en particulier. En premier lieu, comme substitut à l'objet autistique, puis comme support de l'enveloppe individuelle de l'enfant, pour finir comme symbole paternel pour le groupe. 


\section{Première séance : être ensemble quelle violence ?}

Septembre 2002, arrivés dans la salle, j'énonce à tous les règles du groupe. Les enfants découvrent juste ensuite que les tommettes du sol s'enlèvent. Ils commencent à démonter le sol, carreau par carreau. Au début, je verbalise peut-être l'attaque du cadre qu'ils étaient en train d'agir, et, le fait qu'ils n'étaient pas dans le semblant. Puis, tout va très vite, ils m'apportent plus d'une dizaine de carreaux et se sentent excités par ce démontage. Tous ensemble, ils se frappent avec les tommettes, enfreignant la règle énoncée, mais surtout au risque de se blesser. Nous les stoppons et recouvrons la scène d'un tapis pour éviter que la poudre blanche du sol ne soit éparpillée. Mais elle nous recouvre déjà, nous sommes tous blancs.

Nous, les co-thérapeutes, sommes sidérées, ils continuent à prendre les tommettes qu'ils m'ont mises sur les genoux pour se frapper. Je cherche un endroit pour les protéger mais la salle est dépouillée de meubles qui ferment. Je sors alors de la pièce et mets les tomettes dans une corbeille à papier. Le groupe me suit et s'éparpille sur le palier. Je me rends compte... que je viens de transgresser moi aussi une des règles énoncées : on ne doit pas sortir de la pièce. Je leur verbalise cette transgression commune. Me suis-je mise, inconsciemment, au même endroit qu'eux, pour qu'on se trouve dans un passage à l'acte inacceptable?

Tout le monde revient dans la salle et ma cothérapeute commence l'histoire de Cendrillon. Alain déplace sa chaise à côté d'elle, intéressé, Antoine reste loin du groupe allongé par terre en réclamant un grand sachet, Kenny fait tomber la chaise d'Antoine et vient se placer entre nous. L'unité qu'ils venaient de trouver dans le démontage et le pulsionnel a été aussi stoppée par notre incapacité sidérative à transformer ces éléments $\beta$. Par notre intervention, leur ébauche d'un groupe pulsionnel n'a pu se réaliser. Nous n'avons pas accepté leur mode de rassemblement autour de l'objet dans la violence, comme si c'était un objet anti-relationnel.

Alain écoute bien l'histoire et regarde les images. Sa boîte de bonhommes est placée au-dessus de l'armoire, je lui avais assuré que « les objets personnels restent dans la salle et seront rendus à la fin de la séance ». Alain a gardé, malgré tout, un « dingo » qui sera découvert à la fin du groupe bien caché dans sa main. Il est très angoissé car ses bonhommes sont loin, comme s'il était arraché d'une partie de lui. Il veut être Cendrillon, mais ne le fait pas, il dit «j'ai peur » lorsque ma cothérapeute lui demande s'il veut se déguiser, comme s'il allait disparaitre sous le vêtement, fondre dans le rôle. Il tourne autour de ses bonhommes en-haut sur l'armoire. Je lui dis : « On les a mis sur l'armoire car tu ne peux pas prendre d'objets personnels ici... Tu as peur de ne pas les retrouver? » Toute la séance, il touche sa poche en même temps que le coin de la jupe de ma cothérapeute qui lui fera penser à 
un doudou comme s'il voulait rester en lien, rester collé à la fonction maternante.

Kenny, de son côté, regarde les images du livre et pose des questions - qui est qui ? -, même s'il connaît la réponse. Ma cothérapeute confirme une réponse : «C'est la fée. » Il touche le livre, puis elle et moi, comme s'il caressait indifféremment objets et personnes, il n'y a pas d'espace. Il m'embrasse. À tour de rôle, on lui répète les règles du groupe de ne pas se toucher et faire semblant. Il prend le rôle de Cendrillon, met une robe bleue et ne parlera plus dans la séance, il ne poussera que de petits cris. Il me couvre la tête de tous les déguisements et se cache sous la table. Je lui demande : «Tu ne veux plus me voir où tu ne veux plus que je te vois ? »Souvent les patients ne répondaient pas. Plus tard, je comprendrais que c'est l'exclusivité de la relation à l'adulte qu'il recherche, qu'il se sent attaqué par les autres, des bébés rivaux. Il paraît plus calme, mais donne des coups de pieds à Antoine sous la table aussi, il sera sorti de l'espace jeu. Puis, de retour dans le jeu, il fera semblant de dormir avec Antoine. Je dis : «On dirait qu'ici tout le monde veut dormir. » Il arrive ensuite à faire semblant de taper Antoine avec le pouf, puis le sac. Il s'ensuit avec ce sac une recherche d'enveloppement entre intérieur-extérieur, où il y met des vêtements, puis le transforme en chapeau et se regarde dans le miroir.

Antoine n'écoute pas l'histoire. Il est allongé comme répandu sur le tapis et cherche un grand sachet qu'il a toujours avec lui à la main. Il est dans sa poche. À la question «Quel rôle tu veux jouer ? », il répète inlassablement quelque chose d'inaudible «nan shachet». Je saisis le mot et l'indifférenciation qu'il montre, entre avoir un sachet, son objet autistique et être un rôle. Je le considère de la place où il est, un objet, pas un sujet, tout en prenant en compte ce qu'il semble dire, "grand sachet ». Lorsque le bébé vocalise, la mère interprète les sons en leur donnant du sens ; ici, je pars de la même hypothèse, donner du sens dans un lieu contenant aux vocalises des enfants mutiques. Je l'assigne à une place participative en lui disant : "Tu seras M. Grand Sachet », entre vivant et mort, comme s'il devenait son objet autistique, mais avec une identité humaine vers le masculin. Il se déguise alors avec une immense salopette en blue jeans qui est à l'envers, style salopette de garagiste. Elle ressemble à un sac, un grand sachet. Les séances où il la mettra, il enlèvera toujours les siens. Il veut accrocher les bretelles, se met devant moi et, seul, il arrive à mettre un bouton, le dernier. Pour les autres, il se plante devant ma co-thérapeute et dit : « Maman », un terme indifférencié pour les hommes et les femmes. Durant le temps du jeu il reste collé par terre en se tortillant. Il enlève facilement le déguisement à la fin et on comprend deux mots, « peur » et « Kenny » dans la discussion.

Dans cette première séance, les éléments $\beta$, pulsionnels, foisonnent et nous n'arrivons pas à les transformer. Nous les recevons et les gardons en nous. Les patients testent violemment les limites du cadre 
que nous avons du mal à garder. La salopette paraît être une protection contre le groupe pour un enfant.

\section{Synergie sachet/salopette, vivant/mort, enveloppe/à vif}

Dès la deuxième séance, Antoine substitue son sachet plastique au vêtement salopette. Il regarde le sachet plastique de la poubelle, mais ne le prend pas. L'objet autistique hérité d'un pseudo lien familial, de quelque chose du père, se transforme ${ }^{4}$. D'externe, petit, volant, il devient une enveloppe externe du corps. Un déplacement de contenu à contenant s'opère, qui contient quoi. Le sachet est un contenant qui, semblait-il, contenait son angoisse dans la chambre chez son père. Voletant derrière lui contenait-il ses pensées comme un cerveau extra corporel ? Formalisé par la salopette d'homme trop grande dans le jeu, devient-il le contenant d'Antoine comme enveloppe pare-excitative du groupe ? On dirait qu'Antoine est dans un sachet. Comme un ruban de Mœbius, le sachet avait-il une fonction de pare-excitation du père, des pensées? Il reste hors du groupe sur le tapis par terre, face en l'air comme s'il gisait là, mort pendant la lecture de l'histoire de la Chèvre de M. Seguin. Le loup ? Il s'approche. Puis reste à la périphérie du groupe, mais jette des coups d'œil aux autres...

Alain essaie de mettre un gant, Antoine approche et tente d'entrer dans le jeu d'enfiler le gant. Il marche sur les jambes de la salopette, la remonte, mais elle l'entrave. Il râle. Il se regarde dans le miroir, regarde les jambes trop longues. Il tourne dans la pièce, longe les murs. Comparaison insupportable grand/petit, père/fils ? Que cherche-t-il à délimiter : le contenant salle ou le contenu Antoine ? Lui contenu dans la salopette, elle-même contenue dans la salle ? La synergie s'opère entre la salle, la salopette, lui et le psychodrame.

Alain, lui aussi devant le miroir, se regarde à différentes distances. Qui est-il ? Kenny, en Blanche-Neige, essaie d'entrer dans ce jeu spéculaire le narguant avec le deuxième gant. Mais ils n'ont pas la même quête, Kenny cherche la mère en lui.

Dans la séance, les patients projettent leurs angoisses archaïques sur le groupe. Chacun tente de se protéger des attaques de l'autre, du groupe. Et même la salopette contenante peut être vécue comme attaquante.

Les sept séances suivantes, Antoine se déguisera avec la salopette. Le statut de cet objet évoluera dans le groupe indiquant un changement de position psychique chez Antoine et l'émergence de l'intérêt de la fonction paternelle pour le groupe. Il reste dans une position passive de « nan chachet» plusieurs séances. Du sensoriel des premières séances où il rampe caché sous les déguisements ou rasant les murs, il passera à une position verticale participative.

4. Se référer à l'anamnèse pour le sachet plastique. 
Au fil de ces sept séances, il rejettera le sachet plastique à la poubelle que lui tendait Kenny, l'objet autistique ayant perdu son attrait ; il l'a transformé en enveloppe corporelle. Lorsque l'objet salopette sera un jour attaqué par Alain, Antoine l'affrontera, abordant ainsi la position active et la lui arrachera, défendant l'objet non-moi, en la remettant à l'endroit, pouvant agir sur l'objet et le retransformer, lui donner du bon sens. Dans la première séance il avait laissé la salopette à l'envers. L'objet ainsi convoité par un autre du groupe laisse émerger quelque chose d'un désir inconscient d'incorporation puis d'introjection ? Si la salopette contenante est un bon objet pour l'un, alors elle doit l'être pour un autre et le désir de prendre pour soi émerge. Lors d'une autre séance, Alain dira qu'il est le père, qu'il dirige la famille come pour prendre l'attribut masculin de cette salopette. Kenny avec la robe bleue reste la mère. Ils disent à Antoine : «Tu es l'enfant. » Le groupe se structure en un jeu commun de figuration familiale. L'illusion groupale se transforme, chacun prend sa place. Les trois enfants alors se collent par terre les uns sur les autres en poussant des râles. Question de la fusion, l'enveloppe du groupe familiale est-elle une peau pour tous ? La famille, est-ce être fusionné ? Aller-retour entre intérieur et extérieur, c'est quand ils se différencient, défusionnent par les rôles qu'ils fusionnent par le jeu. Antoine se fait marcher dessus par la mère en colère. Ils s'écrasent les uns contre les autres. "Ici on ne peut que se mélanger, s'écraser, il n'y a pas de différence ? », dis-je. Ma cothérapeute rappelle les règles du groupe, « On ne se touche pas », comme si cet amalgame de chairs fusionnées dans le contre-transfert nous était insupportable pour en appeler au cadre.

Antoine reboutonne la salopette et dit à Alain : « Je suis le papa. » Il se confronte au père Alain et rejette la position psychique d'enfant qu'ils lui avaient attribuée. Il affirme son identité du côté du père et se met devant le miroir, il attache sa salopette. Il me regarde et dit «Moi, je respecte les règles. » Nous sommes étonnées de sa capacité à lancer des phrases correctes depuis quelques séances. À partir de là, Antoine ayant testé le rôle du père, acceptera de se confronter aux autres dans des jeux violents. Comme le jeu de faire disparaître le père sous les habits. Alain ne le supporte pas, l'objet ne garde pas sa permanence. Antoine rit très fort, il a compris qu'il ne disparaît pas vraiment. Il aborde enfin des jeux de tout petit devant notre regard bienveillant. Puis il finira la séance couché en foutus recouvert de vêtements. Ma cothérapeute dit : «Le papa se cache ». Il répète en écho «se cache ».

La séance 9 semble marquer la naissance d'Antoine, si l'on peut dire, après neuf séances de gestation. La salopette disparaît du jeu comme si quelque chose de l'enveloppe était intégrée, le feuillet externe du pare-excitation ? Antoine s'autorise à choisir un rôle de « maman » puis de «papa ». Les autres lui disent : «Tu es le papa. » Mais non, il prendra une grenouillère d'adulte qu'il mettra par-dessus ses vêtements, et jouera le bébé. Quelque chose de l'enveloppe pare- 
excitative a été introjectée. La construction psychique du pare-excitation transforme l'adaptation d'Antoine au groupe, il peut garder ses vêtements. Le groupe/famille est ainsi constituée, Alain, le grand garçon, est aujourd'hui le leader et cherche « à faire tomber la maman ». Il entraîne les autres, la mère et le bébé à transgresser les règles et nous force à intervenir physiquement car il monte dangereusement sur l'armoire. Ils en profitent pour s'asseoir sur nos chaises. Je dis : « Ici, c'est la bataille contre les parents, comme s'il devait y avoir un gagnant. » Comme s'il s'agissait de la lutte pour la vie psychique. La cohésion du groupe nous montre un scénario infantile, ils jouent à prendre la place des parents.

\section{Vers l'émergence d'un Moi ?}

Dans les séances suivantes, la cohésion du groupe restera, ils testeront les facettes de la mère, du père et du bébé. Dans la douzième séance, à la suite de constant changement de rôle, Alain finira par dire : «Je redeviens Alain. » Les processus identificatoires ont été mis au travail par le jeu de miroir, de disparition et de réapparition sans en mourir, pour finir par tester les différentes places identitaires du scénario familial dans le jeu psychodramatique.

Je dis : « Chacun ici fait son chemin. » Kenny pose une question fondamentale : «Une maman, c'est comme ça ? » Je renvoie vers le groupe : «Qu'en pensez-vous ? Une maman, c'est comme ça ?» L'écho est corporel : Antoine dort dans son lit, Alain est fasciné par son reflet.

L'absence de Kenny à une séance fera dire à Alain : «C'est Kenny qui l'a dessiné » luttant contre l'absence mais en effaçant le dessin du tableau. Le bébé Antoine ajoutera : « Kenny n'a pas pu faire semblant. » Ils ont construit un scénario familial fantasmatique et les espaces se différencient, entre « jeu et réalité » à chaque séance.

Antoine passe ainsi des processus archaïques, très sensoriels des premières séances, aux processus primaires où il apparaît ensuite comme satisfaisant ses moindres pulsions agressives, pour finir par élaborer un autre niveau de pensée avec l'arrivée des processus secondarisés décrits ci-dessous. Pensée contenue par la fonction $\alpha$ de la co-thérapeute qui lui fera découvrir le verbe, l'action, en un mot la représentation projection/introjection. Dans cette vignette clinique il dit d'une voix monocorde « le bébé boit », elle dit : «Le bébé boit »; il dit «Le bébé jette », elle répète : «Le bébé jette » et ce sur plusieurs phrases. Puis il mime l'action qu'il dit et prend plaisir à faire semblant. L'aller/retour entre eux est stupéfiant. Il va même écrire sur le tableau « travail »! Il prend plaisir à découvrir la pensée, de passif il devient actif. La position de sujet émerge sensiblement de la position d'objet. Dans l'action il peut maintenant se mettre à la place de l'autre et défend 
Alain contre Kenny qui allait le frapper. Il peut ressentir la détresse de l'autre.

Pour la dernière séance en juin, ils ont choisi une histoire où Petit Ours Brun regarde les étoiles avec son père. Antoine la raconte en l'inventant ${ }^{5}$, il décrit les images avec l'intonation de ma cothérapeute quand elle raconte. Kenny prend la suite. Les rôles sont inversés Antoine l'embête.

J'éternue, Antoine me dit : «À tes souhaits, Laurence », puis il éternue. Le mimétisme est parfait. Je dis : « À tes souhaits », il me répond : «Merci. » Quels processus a-t-il incorporés puis introjectés ? Du corps à la pensée, au monde social, les mots de politesse représentent-ils un Surmoi en cours d'intégration ? Introjection de la relation à l'autre, qui, en miroir, exprime du social, venant renforcer ses assisses narcissiques. L'angoisse s'amenuise et laisse place au plaisir d'être en relation.

À la fin du groupe, Alain étale des vêtements d'homme, il aborde aussi quelque chose de la fonction paternelle. Antoine se précipite bruyamment et se roule dessus. Alain remet les habits, enveloppe vide, l'absence d'un père qu'Antoine a peut-être dépassée ?

\section{CONCLUSION}

À partir du transfert agglutiné/symbiotique des premières séances dans lequel se trouvaient nos patients, la capacité de symbolisation a été abordée. Un constant aller-retour de mécanismes projectif et introjectif les a conduits à une différenciation des espaces, créant un Surmoi groupal petit à petit introjecté pour l'un, voir incorporé pour les autres.

La cocréation du lien groupal au travers de différentes enveloppes dont le cadre très particulier a permis aux enfants de tester les différentes places du scénario familial joué en groupe. Ils ont pu alors introjecter une forme de représentation familiale en mettant en jeu des processus identificatoires. Parallèlement, le statut de l'objet pour un patient, s'est transformé d'autistique en contra-phobique, puis en objet de relation.

De la matière corporelle des processus archaïques, le groupe lui a permis de les transformer en processus plus secondarisés. Ainsi la différenciation des espaces entre l'animé et l'inanimé a pu se réaliser.

5. Il ne sait pas encore lire. Il apprendra seul l'année suivante en utilisant l'alphabet comme stéréotypie. 


\section{BIBLIOGRAPHIE}

ANZIEU, D. 1956. Le psychodrame analytique chez l'enfant et l'adolescent, Paris, PUF, 2004.

BIon, W. R. 1967. Recherche sur les petits groupes, Paris, PUF, 2004.

Bion, W. R. 1967. Réflexion faite, Paris, PUF, 1983.

Brun, A. 2007. Médiations thérapeutiques et psychose infantile, Paris, Dunod.

Chouvier, B. et coll. 2002. Matière à symbolisation, Lausanne, Delachaux et Niestlé.

Freud, S. 1929. Malaise dans la culture, Paris, PUF, 1997.

Gimenez, G. 2002. «Les objets de relation », dans B. Chouvier et coll. (sous la direction de), Les processus de la médiation, Paris, Dunod.

HAAG, G. 2005. «Comment les psychanalystes peuvent aider les enfants avec autisme et leur famille », dans B. Golse ; P. Delion (sous la direction de), Autisme. États des lieux et horizons, Toulouse, érès, 2006.

JAITIN, R. et coll. 2010. «Défaut de symbolisation et aménagement des dispositifs dans le groupe thérapeutique d'enfant », Revue française de psychothérapie psychanalytique de groupe $\mathrm{n}^{\circ} 53$, Toulouse, érès, Les groupes pour les enfants, les adolescents et leur famille.

KAËS, R. et coll. 1999. Le psychodrame psychanalytique de groupe, Paris, Dunod.

Kestemberg, E. ; JeAmmet, P. 1987. Le psychodrame psychanalytique, Paris, Puf, «Que sais-je » $\mathrm{n}^{\circ} 2337$. 